\title{
Two-by-two Substitution Systems and the Undecidability of the Domino Problem
}

\author{
Nicolas Ollinger \\ Laboratoire d'informatique fondamentale de Marseille (LIF) \\ Aix-Marseille Université, CNRS, \\ 39 rue Joliot-Curie, 13013 Marseille, France, \\ Nicolas.0llinger@lif .univ-mrs.fr
}

\begin{abstract}
Thanks to a careful study of elementary properties of two-bytwo substitution systems, we give a complete self-contained elementary construction of an aperiodic tile set and sketch how to use this tile set to elementary prove the undecidability of the classical Domino Problem.
\end{abstract}

\section{Introduction}

The Domino Problem is the following simple problem: given a finite set of tiles, copies of the unit square with colored edges, decide if it is possible to tile the whole euclidian plane using as many copies of each tile as you need ensuring that tiles colors match along edges, without scaling or rotating the tiles. This problem was first described by Wang to study a particular syntactical restricting of the Entscheidungsproblem (for a proof of the logical problem without using the Domino Problem and an explanation of the field, see [6]). The Domino Problem turns out to be undecidable, as it was proved in 1964 by Berger [1, 2], a student of Wang. The undecidability of the Domino Problem as since been used outside its original realm, providing a valuable tool to prove undecidability results, see for example the results of Kari [7] on cellular automata.

The historical proof, as found in [1], is very technical. One technical difficulty of the proof is the involvement of aperiodic tile sets: set of tiles that only admit aperiodic tilings. Several authors worked both to ameliorate the proof of the Domino Problem and to construct simpler aperiodic tile sets. The most quoted proof is certainly the one from Robinson [12], the first proof involving substitutions is the one of Mozes [10]. For an historical survey of the field, the reader might consult [5] and [11].

Recently, several authors have been independently interested into providing new proofs of the undecidability of the Domino Problem: Durand, Levin, and Shen [3] revisited the classical Robinson proof technic introducing new tools based on substitutions; Kari [8] proposed a completely new proof technic reducing the Domino Problem to the immortality problem rather than on the halting problem; Durand, Romashchenko, and Shen [4] proposed another new proof technic based on Kleene's fixed point theorem.

In the present paper, we give a complete self-contained elementary construction of an aperiodic tile set by combining tools from $[12,10,3]$ with a careful 
study of two-by-two substitution systems. Our claim is that this proof both explains where the tile set comes from and why it works. Moreover, the number of tiles (104) is a reasonable compromise between classically big tile sets (more than 16000 tiles in $[12,3]$ ) and very involved small tile sets (see [5] for details on the competition). Furthermore, the tile set as a nice property to be easily extendable to code any substitution, leading to a new and shorter proof of the undecidability of the Domino Problem sketched in 4.

\section{Two-by-two Substitution Systems}

A pattern $\mathcal{P}$ is a subset of the discrete plane $\mathbb{Z}^{2}$. The translation $\mathcal{P}+u$ of a pattern $\mathcal{P}$ by a vector $u \in \mathbb{Z}^{2}$ is the pattern $\{z+u \mid z \in \mathcal{P}\}$. Let $\boxplus^{i}$ denote for all $i \in \mathbb{N}$ the pattern $\left\{x \in \mathbb{Z} \mid 0 \leqslant x<2^{i}\right\}^{2}$ : the square of size $2^{i}$ with south-west corner at $\left(\begin{array}{l}0 \\ 0\end{array}\right)$. The two-by-two square $\boxplus^{1}$ is abbreviated as $\boxplus$. The two-by-two scaling $\square(\mathcal{P})$ of a pattern $\mathcal{P}$ is the pattern $\{2 z+c \mid z \in \mathcal{P}, c \in \boxplus\}$.

Let $\Sigma$ denote a finite set, or alphabet. A coloring $\mathcal{C}: \mathcal{P} \rightarrow \Sigma$ is a covering of a pattern $\mathcal{P}$, the support of $\mathcal{C}$ denoted as $\operatorname{Sup}(\mathcal{C})$, by letters of $\Sigma$. A subcoloring $\mathcal{C}^{\prime}$ of a coloring $\mathcal{C}$ is a restriction of this coloring, formally $\mathcal{C}^{\prime}=\mathcal{C}_{\mid \operatorname{Sup}\left(\mathcal{C}^{\prime}\right)}$. The translation $u \cdot \mathcal{C}$ of a coloring $\mathcal{C}$ by a vector $u \in \mathbb{Z}^{2}$ is the coloring with support $\operatorname{Sup}(\mathcal{C})+u$ satisfying $u \cdot \mathcal{C}(z+u)=\mathcal{C}(z)$ for all $z \in \operatorname{Sup}(\mathcal{C})$. A coloring $\mathcal{C}$ occurs in a coloring $\mathcal{C}^{\prime}$, denoted as $\mathcal{C} \prec \mathcal{C}^{\prime}$, if a translation of $\mathcal{C}$ is a subcoloring of $\mathcal{C}^{\prime}$.

A coloring $\mathcal{C}$ is periodic, with period vector $p \in \mathbb{Z}^{2}$, if, for all $z \in \operatorname{Sup}(\mathcal{C})$, if $z+p \in \operatorname{Sup}(\mathcal{C})$ then $\mathcal{C}(z+p)=\mathcal{C}(z)$. An aperiodic coloring is a coloring admiting no non-trivial period (i.e. other than the trivial period 0 ). A set of coloring is aperiodic if it is not empty and all its colorings are aperiodic.

Let $X$ be the set of colorings with support $\mathbb{Z}^{2}$. Endow $X$ with the product topology of the discrete topology on $\Sigma$. This topology is compatible with the metric $d$ defined for all colorings $\mathcal{C}, \mathcal{C}^{\prime} \in X$ by $d\left(\mathcal{C}, \mathcal{C}^{\prime}\right)=2^{-\min \left\{|z|, \mathcal{C}(z) \neq \mathcal{C}^{\prime}(z)\right\}}$. Such topology is compact and perfect. A subset of $X$ both topologically closed and closed by translations is a subshift from symbolic dynamics [9].

A two-by-two substitution system is a pair $(\Sigma, s)$ where $\Sigma$ is a finite alphabet and $s: \Sigma \rightarrow \Sigma^{\boxplus}$ is called the substitution rule. The local rule $s$ is extended to a global rule $S: \Sigma^{\mathcal{P}} \rightarrow \Sigma^{\square(\mathcal{P})}$ mapping colorings into colorings by:

$$
\forall z \in \mathcal{P}, \forall c \in \boxplus, \quad S(\mathcal{C})(2 z+c)=s(\mathcal{C}(z))(c)
$$

The restriction of the global rule to $X$ is a continuous map. The global rule weakly commutes with translations: $S(u \cdot \mathcal{C})=2 u \cdot S(\mathcal{C})$ for all vector $u \in \mathbb{Z}^{2}$ and all coloring $\mathcal{C}$. The $i$-level image of a letter $a \in \Sigma$ by $s$ is the coloring $S^{i}(a)$ with support $\boxplus^{i}$.

Example 1. Figure 1 depicts a variation on the classical chair two-by-two substitution. This substitution will reappear later in this paper. 


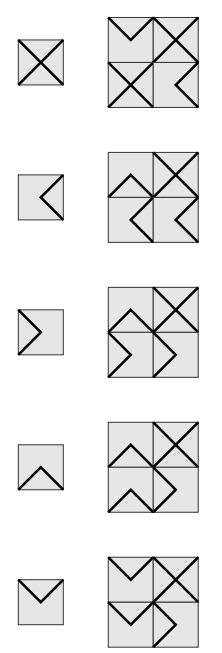

(a) rule $s$

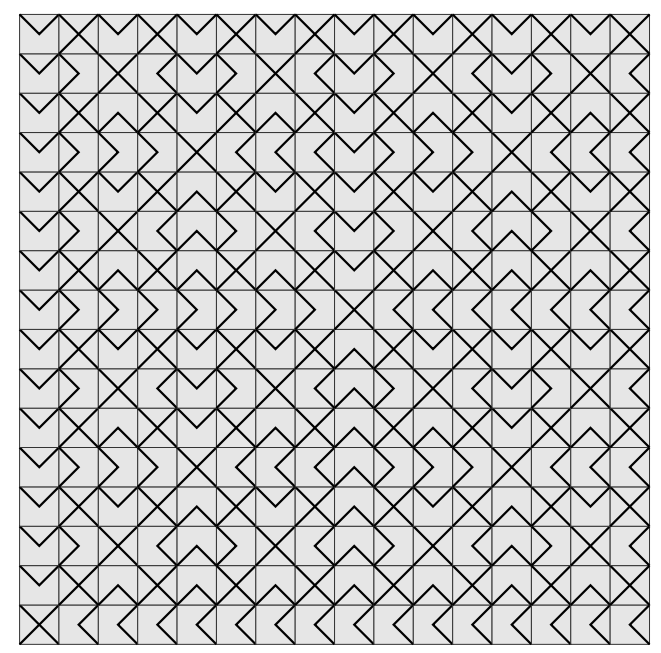

(b) 4-level image $S^{4}(\bigotimes)$

Fig. 1: A sample two-by-two substitution with 5 letters

The literature provides several different methods to extend substitutions to colorings of the whole plane. A classical one is to consider the set $X_{s}$ of colorings such that each of their finite subcolorings $\mathcal{C}$ occurs at some level $i$, that is there exists $a \in \Sigma$ such that $\mathcal{C} \prec S^{i}(a)$.

In this paper ${ }^{1}$, we prefer to take a more dynamical point of view by considering the limit set $\Lambda_{S}$, the intersection $\bigcap_{n \in \mathbb{N}} \Lambda_{S}^{n}$ of a decreasing sequence of nonempty subshifts. Let $\Lambda_{S}^{0}=X$ and $\Lambda_{S}^{n+1}=\left\{u \cdot S(\mathcal{C}) \mid \mathcal{C} \in \Lambda_{s}^{n}, u \in \boxplus\right\}$, for all $n \in \mathbb{N}$. As $S$ weakly commutes with translations, $\Lambda_{S}^{n}$ is precisely the closure by translation of the compact set $S^{n}(X)$. Notice that, depending on $s$, the sets $X_{s}$ and $\Lambda_{S}$ might be different.

Example 2. Consider the constant substitution on two letters $(\{a, b\}, \lambda x . \lambda z \cdot x)$. The set $X_{s}$ contains 2 elements, but $\Lambda_{S}$ contains infinitely many elements (the closure by translation of 16 elementary elements).

An history for a coloring $\mathcal{C} \in X$ is a sequence $\left(\mathcal{C}_{i}, u_{i}\right) \in(X \times \boxplus)^{\mathbb{N}}$ such that $\mathcal{C}_{0}=\mathcal{C}$ and $\mathcal{C}_{i}=u_{i} \cdot S\left(\mathcal{C}_{i+1}\right)$, for all $i \in \mathbb{N}$.

Proposition 1. The set $\Lambda_{S}$ is precisely the set of colorings admitting histories.

Proof. Consider an history $\left(\mathcal{C}_{i}, u_{i}\right) \in(X \times \boxplus)^{\mathbb{N}}$. As $\mathcal{C}_{0}$ is a translation of $S^{i}\left(\mathcal{C}_{i}\right)$ for all $i \in \mathbb{N}$, straightforwardly $\mathcal{C}_{0} \in \Lambda_{S}$. Conversely, let $\mathcal{C}$ be a coloring in $\Lambda_{S}$. By construction, one can find $\left(\mathcal{C}_{i}, u_{i}\right) \in(X \times \boxplus)^{\mathbb{N}}$ such that $\mathcal{C}_{0}=\mathcal{C}$ and $\left(\sum_{j=0}^{i-1} 2^{j} u_{j}\right) \cdot S^{i}\left(\mathcal{C}_{i}\right)=\mathcal{C}$. By compacity of $X$, one can extract from the iterated

\footnotetext{
${ }^{1}$ in fact, even when claiming to construct tilings coding $X_{s}$, all the constructions we know about really code $\Lambda_{S}$ and can do it for all $s$, not only $s$ such that $X_{s}=\Lambda_{S}$.
} 
images by $S$ of subcolorings of the family $\left(\mathcal{C}_{i}\right)$ a new family of colorings $\left(\mathcal{C}_{i}^{\prime}\right)$ such that $\mathcal{C}_{i}^{\prime}=u_{i} \cdot S\left(\mathcal{C}_{i+1}^{\prime}\right)$, for all $i \in \mathbb{N}$.

Informally, to code elements of $\Lambda_{S}$ using only local rules, one can code a whole history and ensure that the validity of the history is locally checkable. One way to achieve that is to ensure that part of the story at each position is locally available. A story simply explains the value of at a particular position according to an history: it is the sequence of substitution rules applied to obtain the value at that particular position.

More formally, the story at position $z \in \mathbb{Z}^{2}$ for an history $\left(\mathcal{C}_{i}, u_{i}\right) \in(X \times \boxplus)^{\mathbb{N}}$ is the sequence $\left(a_{i}, v_{i}\right) \in(\Sigma \times \boxplus)^{\mathbb{N}}$ such that, for all $i \in \mathbb{N}, a_{i}=s\left(a_{i+1}\right)\left(v_{i}\right)$ and $a_{i}=\mathcal{C}_{i}\left(z_{i}\right)$ where $z_{i} \in \mathbb{Z}^{2}$ is the only position such that $z$ is an element of the pattern $\mathcal{P}_{i}=\boxplus^{i}-\sum_{j=0}^{i-1} 2^{j} u_{j}-2^{i} z_{i}$. Notice that the subcoloring of $\mathcal{C}$ of support $\mathcal{P}_{i}$ is a translation of $S^{i}\left(a_{i}\right)$.

Every story is computable from the story of any among two of its four neighbors, most of the time from any of them. Let $\left(a_{i},\left(\begin{array}{l}x_{i} \\ y_{i}\end{array}\right)\right) \in(\Sigma \times \boxplus)^{\mathbb{N}}$ be a story at position $z \in \mathbb{Z}^{2}$, the story $\left(b_{i}, u_{i}\right) \in(\Sigma \times \boxplus)^{\mathbb{N}}$ at position $z+\left(\begin{array}{l}1 \\ 0\end{array}\right)$ can be constructed as follows. Let $k \in \mathbb{N}$ be the smallest $k$ such that $x_{k}=0$. Let $u_{k}=\left(\begin{array}{c}1 \\ y_{k}\end{array}\right)$. For all $i<k$, let $u_{i}=\left(\begin{array}{c}0 \\ y_{i}\end{array}\right)$. For all $i>k$, let $u_{i}=\left(\begin{array}{l}x_{i} \\ y_{i}\end{array}\right)$ and $b_{i}=a_{i}$. For all $i \leqslant k$ let $b_{i}$ be such that $b_{i}=s\left(b_{i+1}\right)\left(u_{i}\right)$. This procedure will always produce the story for position $z+\left(\begin{array}{l}1 \\ 0\end{array}\right)$, but when $k$ is not defined. Stories at positions $z+\left\{-\left(\begin{array}{l}1 \\ 0\end{array}\right),\left(\begin{array}{l}0 \\ 1\end{array}\right),-\left(\begin{array}{l}0 \\ 1\end{array}\right)\right\}$ can be defined symmetrically.

Proposition 2. Every history can be reconstructed from 1,2, or 4 of its stories.

Proof. By construction, an history is completely defined by the set of all its stories. Consider any story $\left(a_{i},\left(\begin{array}{l}x_{i} \\ y_{i}\end{array}\right)\right)$ of a given history. Four different cases may occur depending on both sequences $\left(x_{i}\right)$ and $\left(y_{i}\right)$. If none of them is ultimately constant, the history can be reconstructed by reconstructing the stories of each position of the plane. If exactly one of them is ultimately constant, only half a plane of stories can be reconstructed and two different stories, with different ultimate constants, are needed. If both sequences are ultimately constant, only a quarter of the plane of stories can be reconstructed and four different stories, with different ultimate constants, are needed.

A substitution is aperiodic if its limit set $\Lambda_{S}$ is aperiodic. A substitution is unambiguous if, for every coloring $\mathcal{C}$ from its limit set $\Lambda_{S}$, there exists a unique coloring $\mathcal{C}^{\prime}$ and a unique translation $u \in \boxplus$ satisfying $\mathcal{C}=u \cdot S\left(\mathcal{C}^{\prime}\right)$. Notice that the injectivity of the local rule is not sufficient to enforce unambiguity. Every unambiguous substitution admits a unique history.

Proposition 3. Every unambiguous substitution is aperiodic.

Proof. Let $s$ be an unambiguous substitution. Let us assume that $s$ is not aperiodic. Let $p$ be the smallest non-trivial period of a coloring in the limit set of $s$ for 
the maximum norm. Let $\mathcal{C} \in \Lambda_{S}$ be $p$-periodic. Let $u$ and $\mathcal{C}^{\prime}$ satisfy $\mathcal{C}=u \cdot S\left(\mathcal{C}^{\prime}\right)$. By construction, $(p+u) \cdot S\left(\mathcal{C}^{\prime}\right)$ is equal to $\mathcal{C}$. As $s$ is unambiguous, $p$ has to be a multiple of $2, p=2 p^{\prime}$, so that $(p+u) \cdot S\left(\mathcal{C}^{\prime}\right)=u \cdot S\left(p^{\prime} \cdot \mathcal{C}^{\prime}\right)$ and $\mathcal{C}^{\prime}=p^{\prime} \cdot \mathcal{C}^{\prime}$. Thus $\mathcal{C}^{\prime} \in \Lambda_{S}$ is $p^{\prime}$-periodic and $p^{\prime}$ is smaller than $p$, contradicting our hypothesis.

Example 3. The substitution on Figure 1 is unambiguous thus aperiodic.

A syntactical way to enforce unambiguity of a substitution $s$, as used in [3], is to ensure that it is injective and that one of the four projectors $s_{i}: a \mapsto s(a)(i)$ has an image disjoined from the images of the other three.

\section{Tilings}

A domino relation $\mathcal{R} \subseteq Y \times Y$ over a finite set $Y$ satisfies the domino property :

$$
\forall a, b, c, d \in Y^{4}, \quad a \mathcal{R} c \wedge a \mathcal{R} d \wedge b \mathcal{R} d \rightarrow b \mathcal{R} c
$$

The color set associated to a domino relation $\mathcal{R}$ is the set of equivalence classes of the equivalence relation $\sim_{\mathcal{R}}$ defined on $Y^{2}$ for all $a, b, c, d \in Y$ by $(a, c) \sim_{\mathcal{R}}(b, d)$ if $a \mathcal{R} c \wedge a \mathcal{R} d \wedge b \mathcal{R} d$. The right color of an element $a \in Y$ is the color $|a\rangle$ such that there exists $b$ satisfying $(a, b) \sim_{\mathcal{R}}|a\rangle$. Symmetrically, the left color of an element $b \in Y$ is the color $\langle b|$ satisfying $(a, b) \sim_{\mathcal{R}}\langle b|$. Straightforwardly, for all $a, b \in Y, a \mathcal{R} b$ if and only $|a\rangle=\langle b|$.

Tilings correspond to the extension of subshifts of finite type (SFT) [9] to $\mathbb{Z}^{2}$ : colorings of the plane satisfying a finite set of local constraints. Several definitions of tiling constraints are possible. In this paper, we focus on so called Wang tiles, but instead of using the classical definition by colors, we use domino relations to simplify the discussions. A tile set $\tau$ is a triple $(T, \mathcal{H}, \mathcal{V})$ where $T$ is a finite alphabet of tiles, $\mathcal{H}$ and $\mathcal{V}$ are domino relations over $T$. A tile set is degenerated if two tiles $a, b \in T$ define the same quadruple of colors $\left(|a\rangle_{\mathcal{V}},|a\rangle_{\mathcal{H}},\left\langle\left. a\right|_{\mathcal{V}},\left\langle\left. a\right|_{\mathcal{H}}\right)\right.\right.$ and $\left(|b\rangle_{\mathcal{V}},|b\rangle_{\mathcal{H}},\left\langle\left. b\right|_{\mathcal{V}},\left\langle\left. b\right|_{\mathcal{H}}\right)\right.\right.$. A pair of tiles $(a, b) \in T^{2}$ matches horizontally if $a \mathcal{H} b$, matches vertically if $a \mathcal{V} b$. A tiling $\mathcal{T} \in X$ is a coloring satisfying the tiling constraints: for all $z \in \mathbb{Z}^{2}$, the pair $\left(\mathcal{T}(z), \mathcal{T}\left(z+\left(\begin{array}{l}1 \\ 0\end{array}\right)\right)\right)$ matches horizontally and the pair $\left(\mathcal{T}(z), \mathcal{T}\left(z+\left(\begin{array}{l}0 \\ 1\end{array}\right)\right)\right)$ matches vertically. The set of tilings $X_{\tau}$ of a tile set $\tau$ is a subshift (of finite type). A tile set is aperiodic if its set of tilings is aperiodic.

Example 4. The tile set $\tau_{0}=\left(\boxplus,\left\{(u, v)|| v-u \mid=\left(\begin{array}{l}1 \\ 0\end{array}\right)\right\},\left\{(u, v)|| v-u \mid=\left(\begin{array}{l}0 \\ 1\end{array}\right)\right\}\right)$ admits 4 tilings: the limit set of the simple substitution $\lambda x . \lambda z . z$.

A tile set $\left(T^{\prime}, \mathcal{H}^{\prime}, \mathcal{V}^{\prime}\right)$ codes a tile set $(T, \mathcal{H}, \mathcal{V})$, according to a coding rule $t: T \rightarrow T^{\prime \boxplus}$ if $t$ is injective and $X_{\tau^{\prime}}=\left\{u \cdot t(\mathcal{C}) \mid \mathcal{C} \in X_{\tau}, u \in \boxplus\right\}$.

Example 5. A simple coding scheme is depicted on figure 2. Given a tile set $(T, \mathcal{H}, \mathcal{V})$, the coding tile set is constructed as a layered tile set : a synchronized product of several layers, upper layers being constrained by lower layers. Layer 1 
is the tile set from example 4. Layer 2 is constrained according to layer 1 : on top of $\left(\begin{array}{l}0 \\ 0\end{array}\right)$ stack elements of $T$; on top of $\left(\begin{array}{l}1 \\ 0\end{array}\right)$ stack elements of $\mathcal{H} / \sim_{\mathcal{H}}$; on top of $\left(\begin{array}{l}0 \\ 1\end{array}\right)$ stack elements of $\mathcal{V} / \sim_{\mathcal{V}}$; on top of $\left(\begin{array}{l}1 \\ 1\end{array}\right)$ is an empty element. The matching rules for layer 2 are simple : a tile in $T$ is required to match horizontal and vertical colors of its four neighbor tiles thus propagating its colors to the next $\left(\begin{array}{l}0 \\ 0\end{array}\right)$ tile. The depicted coding rule $t$ maps every tile $a$ as follows. On layer $1, t(a)$ is the identity map. On layer $2, t(a)$ puts $a$ on top of $\left(\begin{array}{l}0 \\ 0\end{array}\right),|a\rangle_{\mathcal{H}}$ on top of $\left(\begin{array}{l}1 \\ 0\end{array}\right)$ and $|a\rangle_{\mathcal{V}}$ on top of $\left(\begin{array}{l}0 \\ 1\end{array}\right)$.

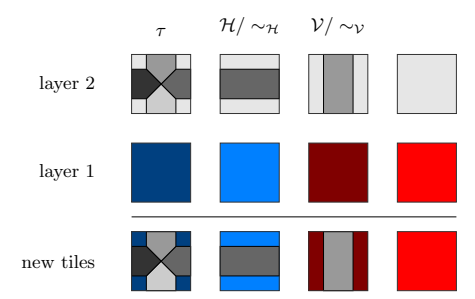

(a) coding tile set

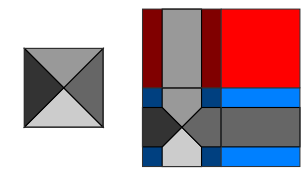

(b) coding rule

Fig. 2: A sample coding scheme

A tile set $(T, \mathcal{H}, \mathcal{V})$ codes a substitution $s: T \rightarrow T^{\boxplus}$ if it codes itself according to the coding rule $s$.

Proposition 4. A tile set both admitting a tiling and coding an unambiguous substitution is aperiodic.

Proof. Let $(T, \mathcal{H}, \mathcal{V})$ be a tile set admitting a tiling and coding an unambiguous substitution $s: T \rightarrow T^{\boxplus}$. By construction, every tiling is the translated image of a tiling by $s$, thus $X_{\tau} \subseteq \Lambda_{S}$. As $X_{\tau}$ is not empty and $\Lambda_{S}$ is aperiodic, $X_{\tau}$ is aperiodic.

\section{An Aperiodic Tile Set of 104 Tiles}

To apply proposition 4 , we construct a fixed point ${ }^{2}$ of a coding scheme in the spirit of the one described in example 5 (this particular coding scheme will not help: the new tile set is always strictly larger than the original one).

One can refine the scheme, as depicted on Figure 3: as the coding scheme generates a layered tile set, one might forget about layer 1 on top of $\left(\begin{array}{l}0 \\ 0\end{array}\right)$ and code it all around on wires in $\left(\begin{array}{l}1 \\ 0\end{array}\right),\left(\begin{array}{l}0 \\ 1\end{array}\right)$, and $\left(\begin{array}{l}1 \\ 1\end{array}\right)$. On top of $\left(\begin{array}{l}1 \\ 1\end{array}\right), 4$ different corners can occur corresponding to the 4 different corners in $\tau_{0}$. On top of $\left(\begin{array}{l}0 \\ 1\end{array}\right), 4$ possible pairs

\footnotetext{
${ }^{2}$ the way we use fixed points with tilings in this paper, whereas sharing similarities with the approach in [4], is less sophisticated.
} 
of wires propagates vertically crossing the $\mathcal{H}$-colors that propagates horizontally. The case of $\left(\begin{array}{l}1 \\ 0\end{array}\right)$ is symmetrical. The new matching rule on layer 2 still requires to match colors but also wires between $\left(\begin{array}{l}1 \\ 1\end{array}\right)$ and its neighbors.

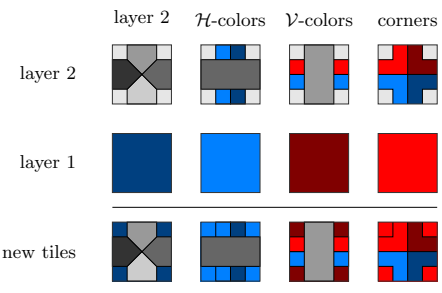

(a) coding tile set

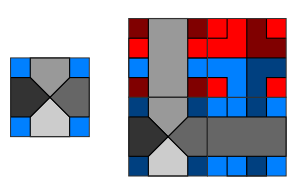

(b) coding rule

Fig. 3: A second coding scheme

A priori, this new coding scheme cannot be applied to every tile set. In a coded tiling, each tile is coded both by its layer 2 component on top of $\left(\begin{array}{l}0 \\ 0\end{array}\right)$ and its layer 1 component by a square wire around neighbors tiles. The only constraints between layer 1 and layer 2 values are checked on $\left(\begin{array}{l}0 \\ 1\end{array}\right)$ and $\left(\begin{array}{l}1 \\ 0\end{array}\right)$ restricting possible $\mathcal{H}$-colors and $\mathcal{V}$-colors for a given layer 1 tile. The coding scheme can only be applied to tile sets closed with respect to this property: if a layer 2 tile $a_{2}$ exists and its four $\mathcal{H}$-colors and $\mathcal{V}$-colors are compatible with a given layer 1 tile $a_{1}$, the tile $\left(a_{1}, a_{2}\right)$ occurs in the tile set.

The new coding scheme cannot be iterated: the coded tile set does not satisfy the closure hypothesis. This can be corrected by adding one bit of information on the wire pair edges of each tile to indicate on which side of the edge one can find the nearest corner: on top of $\left(\begin{array}{l}1 \\ 1\end{array}\right)$ corners are always inside; on top of $\left(\begin{array}{l}1 \\ 0\end{array}\right)$, the nearest corners are outside for both vertical edges; on top of $\left(\begin{array}{l}0 \\ 1\end{array}\right)$, the nearest corners are outside for both horizontal edges. The matching rule has to enforce that both sides of a wire pair edge agree on the direction. With these new bits of information, the coding scheme preserves the closure property and can be iterated. Moreover, it is not strictly increasing and admits a fixed point: a tile set $\tau$ of 104 tiles, the coding substitution of which is depicted on figure 4 . The bits on the edges are considered as a third layer, an inside edge being represented by a $\mathrm{V}$ shape pointing the center of the tile: exactly five tiles occur on layer 3 , the letters of figure 1 .

Before proving the aperiodicity of $\tau$, let us first describe it more precisely. The tile set has three layers. Layer 1 is $\tau_{0}$. Layer 2 consists of $\mathbf{X}, \mathrm{H}$ and $\mathrm{V}$ tiles (see below) transmitting pairs of wires from edge to edge, each wire being colored by an element of $\tau_{0}$ so that on edges, only pairs satisfying $\mathcal{H}_{\tau_{0}}$ and $\mathcal{V}_{\tau_{0}}$ are valid. The matching rule on layer 2 is to match the colors of the facing wires. Layer 3 consists of the five letters from figure 1 . The matching rule on layer 3 is to agree on direction (exactly one $\mathrm{V}$ shape along each edge pointing in one of the two directions). Only the following synchronizations between layers occur inside $\tau$ : 


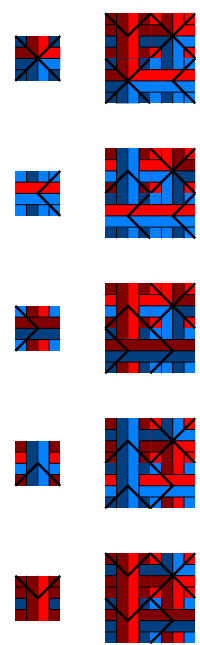

(a) principle

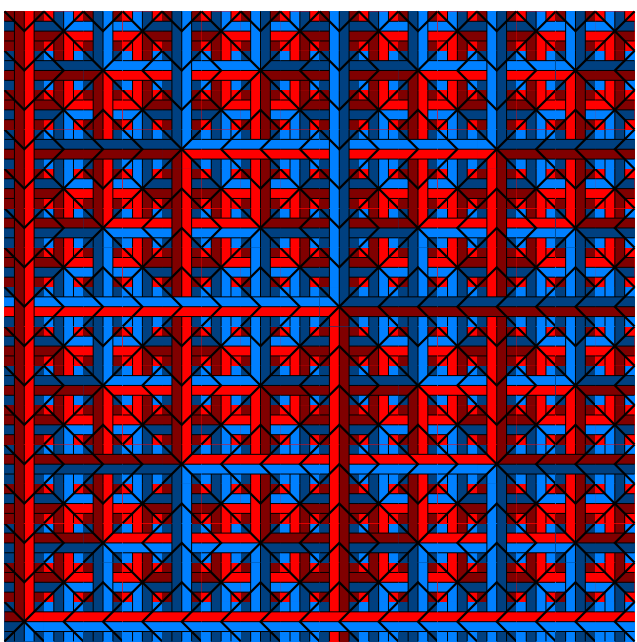

(b) 4-level image $S^{4}$

Fig. 4: a 104 tiles aperiodic tile set $\tau$ coding an unambiguous substitution

$8 \times$ tiles. layer 2 with layer $1\left(\begin{array}{l}0 \\ 0\end{array}\right)$ or $\left(\begin{array}{l}1 \\ 1\end{array}\right)$ and layer 3 equal to $\bigotimes$. $48 \mathrm{H}$ tiles. layer 2 with layer $1\left(\begin{array}{l}0 \\ 0\end{array}\right)$ or $\left(\begin{array}{l}1 \\ 0\end{array}\right)$ and layer 3 equal to $\langle$ or $\rangle$. $48 \mathrm{~V}$ tiles. layer 2 䀯 with layer $1\left(\begin{array}{l}0 \\ 0\end{array}\right)$ or $\left(\begin{array}{l}0 \\ 1\end{array}\right)$ and layer 3 equal to $\triangle$ or $\checkmark$.

There is only 48 tiles of type $\mathrm{H}$ and $\mathrm{V}$ (instead of 64 ) because the propagating direction of their layer 3 has to satisfy the wire color constraint: if one of the orthogonal wires is colored $\left(\begin{array}{l}1 \\ 1\end{array}\right)$, the direction has to point inside the $\left(\begin{array}{l}1 \\ 1\end{array}\right)$ boundary.

The associated substitution rule $s$ transforms a tile $a$ as follows. In position $\left(\begin{array}{l}0 \\ 0\end{array}\right)$, layer 1 is $\left(\begin{array}{l}0 \\ 0\end{array}\right)$ and layers 2 and 3 are the same as for $a$. In position $\left(\begin{array}{l}1 \\ 1\end{array}\right)$, there is an $\mathrm{X}$ tile with layer $1\left(\begin{array}{l}0 \\ 0\end{array}\right)$ and bottom-left wire color equal to the layer 1 of $a$. In position $\left(\begin{array}{l}1 \\ 0\end{array}\right)$, there is an $\mathrm{H}$ tile with layer $1\left(\begin{array}{l}1 \\ 0\end{array}\right)$ : it propagates the wire colors of both its neighbors and points in the same direction as the right edge of the layer 3 of $a$. In position $\left(\begin{array}{l}0 \\ 1\end{array}\right)$, there is a $\bigvee$ tile with layer $1\left(\begin{array}{l}0 \\ 1\end{array}\right)$ : it propagates the wire colors of both its neighbors and points in the same direction as the top edge of the layer 3 of $a$. Notice that the image of a tile is always defined as the wire color constraint is always satisfied.

Theorem 1. The tile set $\tau$ is aperiodic.

Proof. The tile set $\tau$ admits at least one tiling. Consider the substitution $s$ : the four inside edges of each image of a tile by the substitution satisfy the matching rules. Moreover, if two tiles $a$ and $b$ match either horizontally or vertically, $s(a)$ and $s(b)$ will still match in the same direction. Therefore, $\Lambda_{S} \cap X_{\tau} \neq \emptyset$.

The substitution $s$ is unambiguous: it is clearly injective and all the $s_{i}$ projectors have disjoined images. 
The tile set $\tau$ codes $s$. Let $\mathcal{T}$ be a tiling of $\tau$. As layer 1 admits only 4 tilings, there exists $u \in \boxplus$ such that, for all $v \in \boxplus, u \cdot \mathcal{T}(v)$ has layer $1 v$. Thus, $u \cdot \mathcal{T}$ has an $\mathrm{X}$ tile in $\left(\begin{array}{l}1 \\ 1\end{array}\right)$, an $\mathrm{H}$ tile in $\left(\begin{array}{l}1 \\ 0\end{array}\right)$, and a $\mathrm{V}$ tile in $\left(\begin{array}{l}0 \\ 1\end{array}\right)$. Thanks to the wire color constraint, $u \cdot \mathcal{T}_{\mid \boxplus}$ has to be the image of a tile by $s$. Repeating the argument on the $2 \mathbb{Z}^{2}$ translations of $\mathcal{T}$, one conclude that $\mathcal{T}$ is the translated image of coloring by $s$. Moreover, this coloring is a valid tiling: as the information is propagated on layer 2 and 3 by $\mathrm{H}$ and $\mathrm{V}$ tiles, if two tile images $s(a)$ and $s(b)$ match, then $a$ and $b$ match. Therefore, as the image of a tiling by $s$ is also a tiling, $X_{\tau}=\left\{u \cdot s(\mathcal{C}) \mid \mathcal{C} \in X_{\tau}, u \in \boxplus\right\}$.

As $\tau$ admits a tiling and codes an unambiguous substitution, it is aperiodic.

The tile set $\tau$ somehow uses its layers 1 and 2 to draw infinitely many infinite grids to code an history of the substitution of figure 1: the set of layer 3 of tilings is the limit set of the chair substitution. When comparing $\tau$ to aperiodic tile sets of the literature, the author found the following facts. The $\mathrm{PhD}$ dissertation of Berger [1] contains an aperiodic 104 tile set not found in the AMS memoir [2]. Berger's tile set also has three layers, the first two being isomorphic to the one used here... but the third layer is different leading to a more delicate proof and a different set of tilings! Let now $\tau^{\prime}$ be the modified tile set where the colors $\left(\begin{array}{l}0 \\ 0\end{array}\right)$, $\left(\begin{array}{l}1 \\ 0\end{array}\right)$ and $\left(\begin{array}{l}0 \\ 1\end{array}\right)$ are merged in one unique color. The tile set $\tau^{\prime}$ admits more tilings than just the repainted tilings of $\tau$, it has 56 tiles: it is the tile set of Robinson from [12], the aperiodicity proof of which is rather technical.

\section{Enforcing any Substitution}

The tile set $\tau$ might be slightly modified to enforce the limit set of any substitution system $s^{\prime}$ : the idea is to use $\left(\begin{array}{l}1 \\ 1\end{array}\right)$ squares of all sizes to propagate the history of an element of $\Lambda_{S^{\prime}}$. The transformed tile set $\tau\left(s^{\prime}\right)$ is constructed from $\tau$ by replacing $\boxplus$ with $\boxplus \times \Sigma$ on layer 1 and on the wires of layer 2 . The matching rule is extended so that letters have to be equal on layer 1 on the four edges between $\left(\begin{array}{l}0 \\ 0\end{array}\right)$ and $\left(\begin{array}{l}1 \\ 0\end{array}\right),\left(\begin{array}{l}0 \\ 0\end{array}\right)$ and $\left(\begin{array}{l}0 \\ 1\end{array}\right),\left(\begin{array}{l}1 \\ 0\end{array}\right)$ and $\left(\begin{array}{l}1 \\ 1\end{array}\right)$, and $\left(\begin{array}{l}0 \\ 1\end{array}\right)$ and $\left(\begin{array}{l}1 \\ 1\end{array}\right)$. Then, both $\mathrm{X}$ tile and $\mathrm{V}$ tile wire colors are constrained. For any $\mathbf{X}$ tile, let $a$ be the letter on the $\left(\begin{array}{l}1 \\ 1\end{array}\right)$ wire and $b$ be the letter on layer 1 , the constraint is $b=s^{\prime}(a)(u)$ where $u$ depends on the position of the $\left(\begin{array}{l}1 \\ 1\end{array}\right)$ wire $\left(\left(\begin{array}{l}0 \\ 0\end{array}\right)\right.$ for top-right, $\left(\begin{array}{l}1 \\ 0\end{array}\right)$ for bottom-right, $\left(\begin{array}{l}0 \\ 1\end{array}\right)$ for top-left, $\left(\begin{array}{l}1 \\ 1\end{array}\right)$ for bottom-left). For any $\vee$ tile with two $\left(\begin{array}{l}1 \\ 1\end{array}\right)$ wire, let $a$ be the letter on the vertical $\left(\begin{array}{l}1 \\ 1\end{array}\right)$ wire and $b$ the letter on the horizontal one, the constraint is $b=s^{\prime}(a)(u)$ where $u=\left(\begin{array}{l}x \\ y\end{array}\right)$ depends on the positions of both wires $(x=0$ for right, $x=1$ for left, $y=0$ for bottom, $y=1$ for top). Let $\pi$ map every tile of $\tau\left(s^{\prime}\right)$ to $s^{\prime}(a)(u)$ where $a$ and $u$ are the letter and the value of $\boxplus$ on layer 1 .

Theorem 2. Let $s^{\prime}$ be any substitution system. The tile set $\tau\left(s^{\prime}\right)$ enforces $s^{\prime}$ : $\pi\left(X_{\tau\left(s^{\prime}\right)}\right)=\Lambda_{S^{\prime}}$.

Sketch of the proof. Every tiling of $\tau\left(s^{\prime}\right)$ codes an history of $S^{\prime}$ and every history of $S^{\prime}$ can be encoded into a tiling of $\tau\left(s^{\prime}\right)$. 
Corollary 1 (Berger, $1964[\mathbf{1}]$ ). The Domino Problem is undecidable.

Sketch of the proof. Consider the following 6 letters substitution $\mathfrak{s}$ :

$$
T \mapsto \begin{array}{ll}
t & t \\
t & t
\end{array} \quad V \mapsto \begin{array}{ll}
v & v \\
v & v
\end{array} \quad H \mapsto \begin{array}{ll}
h & h \\
h & h
\end{array} \quad t \mapsto \begin{aligned}
& T V \\
& H
\end{aligned} \quad v \mapsto \begin{aligned}
& T V \\
& H V
\end{aligned} \quad h \mapsto \begin{aligned}
& T V \\
& H H
\end{aligned}
$$

Consider the letter $T$ as a place for a tile $\&$, the letter $H$ as a horizontal color transmission path and the letter $V$ as a vertical color transmission path Every coloring $\mathcal{C}$ of the limit set $\Lambda_{\mathfrak{S}}$ containing letters $H, V, T$ has the following property: for all $i \in \mathbb{N}$, a $\boxplus^{i}$ square of $T$ letters, bordered horizontally by $V$ letters and vertically by $H$ letters, eventually spaced by $H$ and $V$ letters providing color transmission, occurs in $\mathcal{C}$. To prove the undecidability of the Domino Problem, recursively construct for every Turing machine a tile set with two layers: on layer 1 put $\tau(\mathfrak{s})$ with the additional constraint that it has only $H, V, T$ letters on layer 1 ; on layer 2 put tiles simulating Turing machine computations on $T$ tiles, so that the bottom left corner of each square (a $T$ connected to a $V$ on the left and the $H$ on the bottom) contains the initialization of the Turing computation. This tile set tiles the plane if and only if the machine does not halt starting from the empty string.

\section{References}

[1] R. Berger, The Undecidability of the Domino Problem, Ph.D. thesis, Harvard University, July 1964.

[2] R. Berger, The undecidability of the domino problem, Memoirs American Mathematical Society, 66(1966).

[3] B. Durand, L. Levin, and A. Shen, Local rules and global order, or aperiodic tilings, Math. Intelligencer, 27(2005), no. 1, 64-68.

[4] B. Durand, A. Romashchenko, and A. Shen, Fixed point and aperiodic tilings, 2007, preprint.

[5] B. Grünbaum and G. C. Shephard, Tilings and patterns, A Series of Books in the Mathematical Sciences, W. H. Freeman and Company, New York, 1989, an introduction.

[6] A. S. Kahr, E. F. Moore, and H. Wang, Entscheidungsproblem reduced to the $\forall \exists \forall$ case, Proc. Natl. Acad. Science, 48(1962), no. 3, 365-377.

[7] J. Kari, The nilpotency problem of one-dimensional cellular automata, SIAM J. Comput., 21(1992), no. 3, 571-586.

[8] J. Kari, The tiling problem revisited (extended abstract), in $M C U$ 2007, volume 4664 of $L N C S$, pages 72-79, Springer, 2007.

[9] D. Lind and B. Marcus, An introduction to symbolic dynamics and coding, Cambridge University Press, Cambridge, 1995.

[10] S. Mozes, Tilings, substitution systems and dynamical systems generated by them, J. Analyse Math., 53(1989), 139-186.

[11] C. Radin, Miles of tiles, volume 1 of Student Mathematical Library, American Mathematical Society, Providence, RI, 1999.

[12] R. M. Robinson, Undecidability and nonperiodicity for tilings of the plane, Inventiones Mathematicae, 12(1971), 177-209. 


\section{A More pictures!}

You will find here some more color pictures. Unfortunately, the construction is very geometrical and colorful. This paper is a compromise between geometrical construction and formal details.

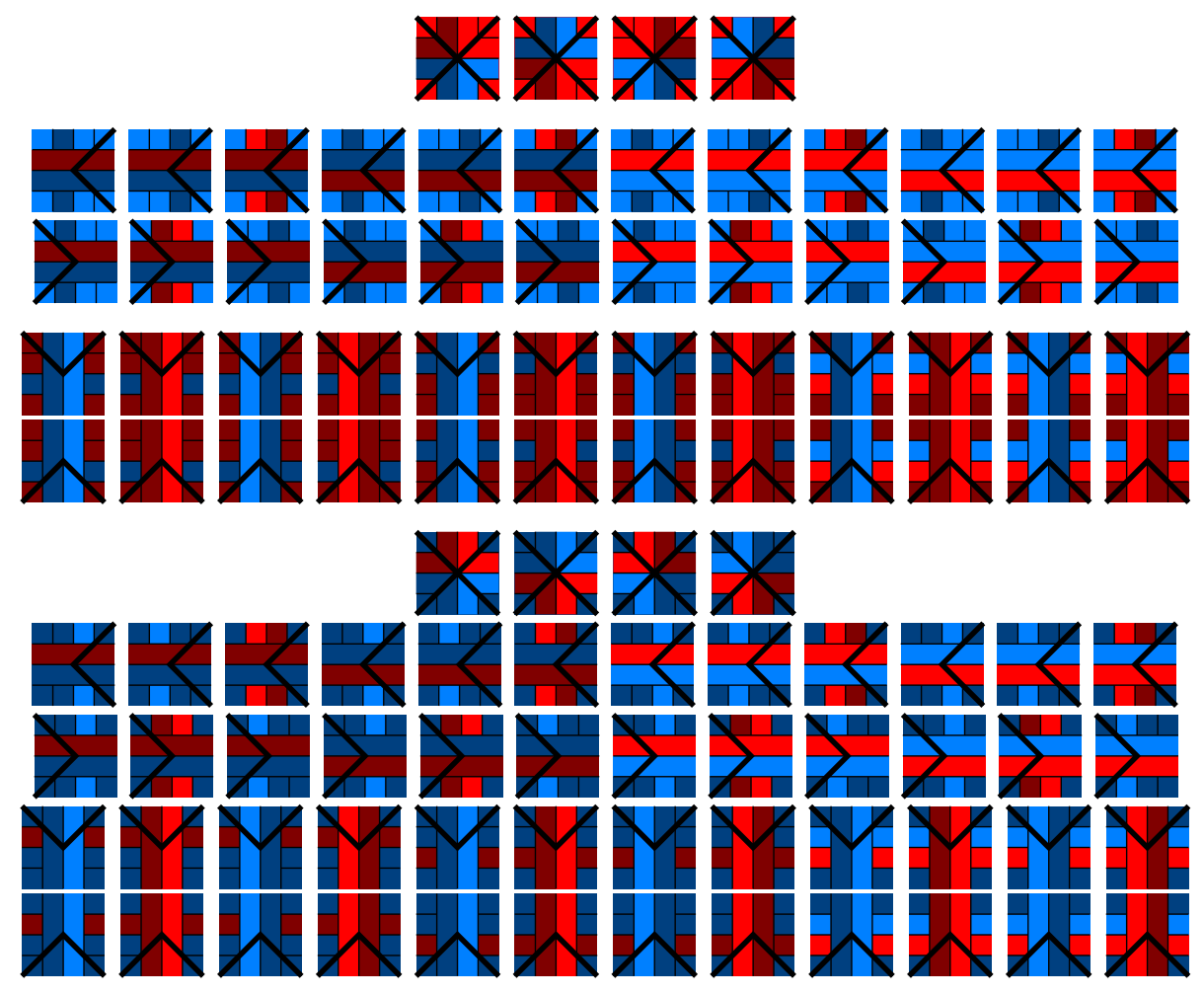

Fig. 5: The 104 tiles of $\tau$ 


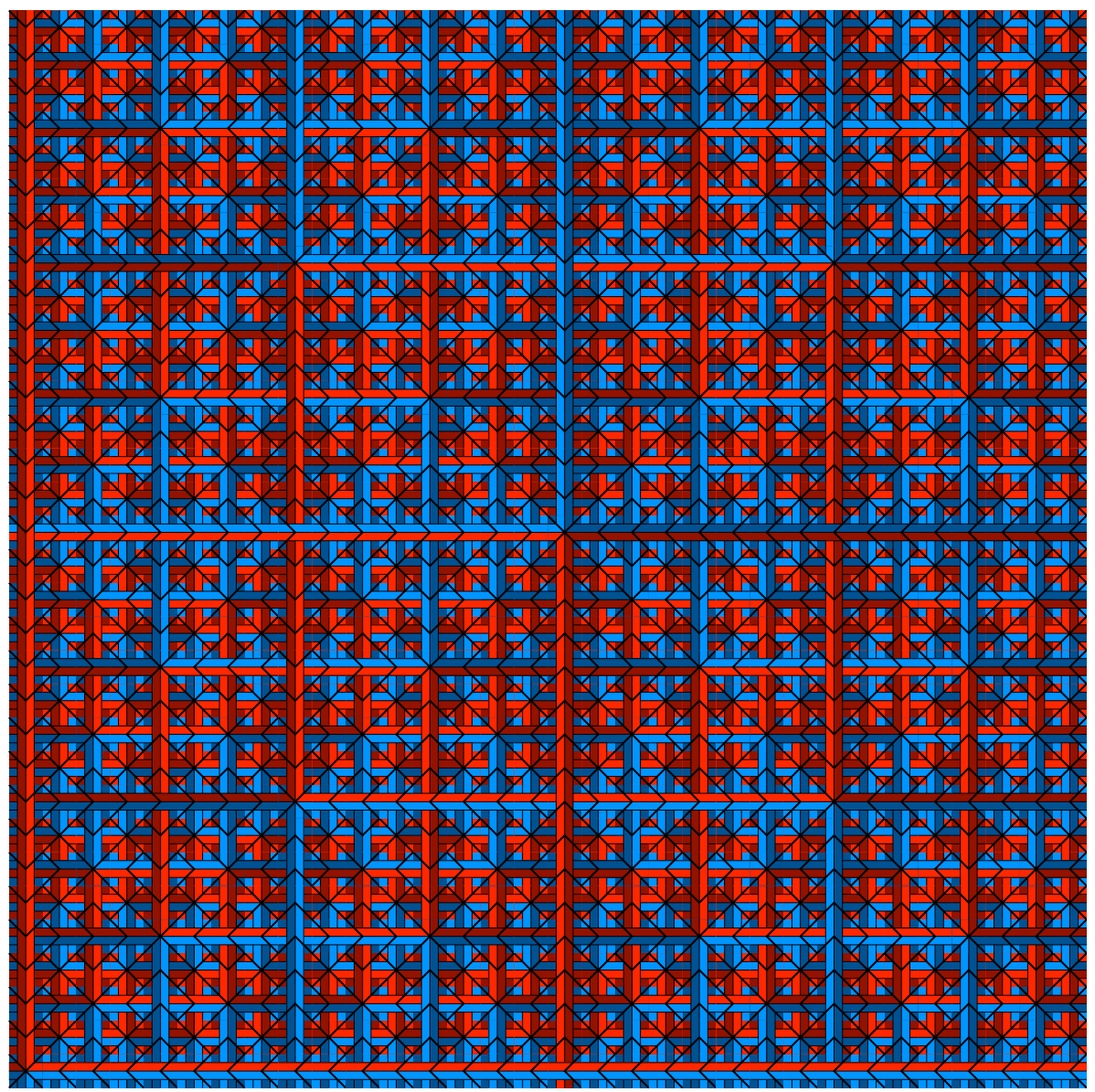

Fig. 6: Part of a tiling by $\tau$ 


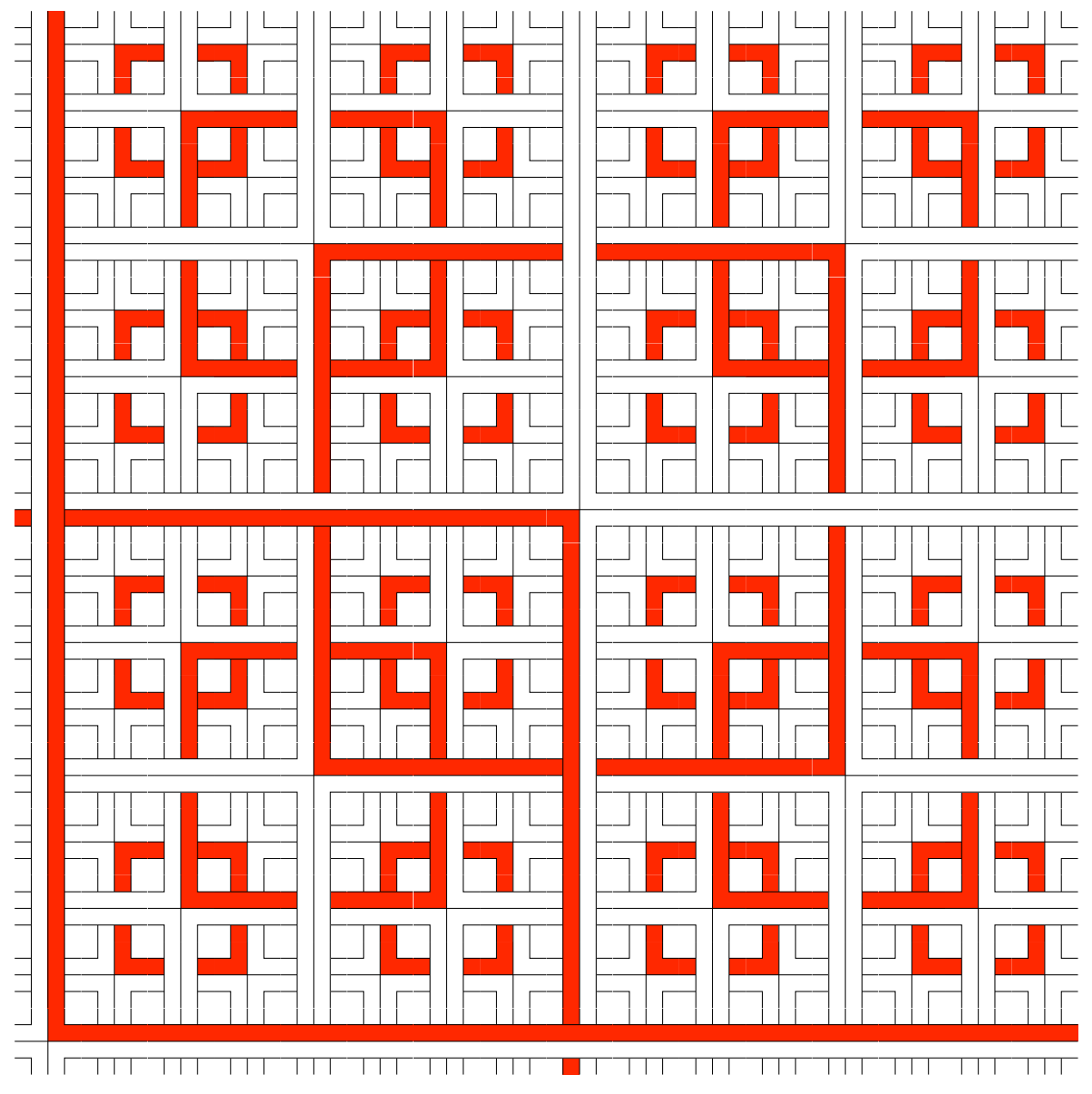

Fig. 7: The $\left(\begin{array}{l}1 \\ 1\end{array}\right)$ squares used to enforce substitutions 

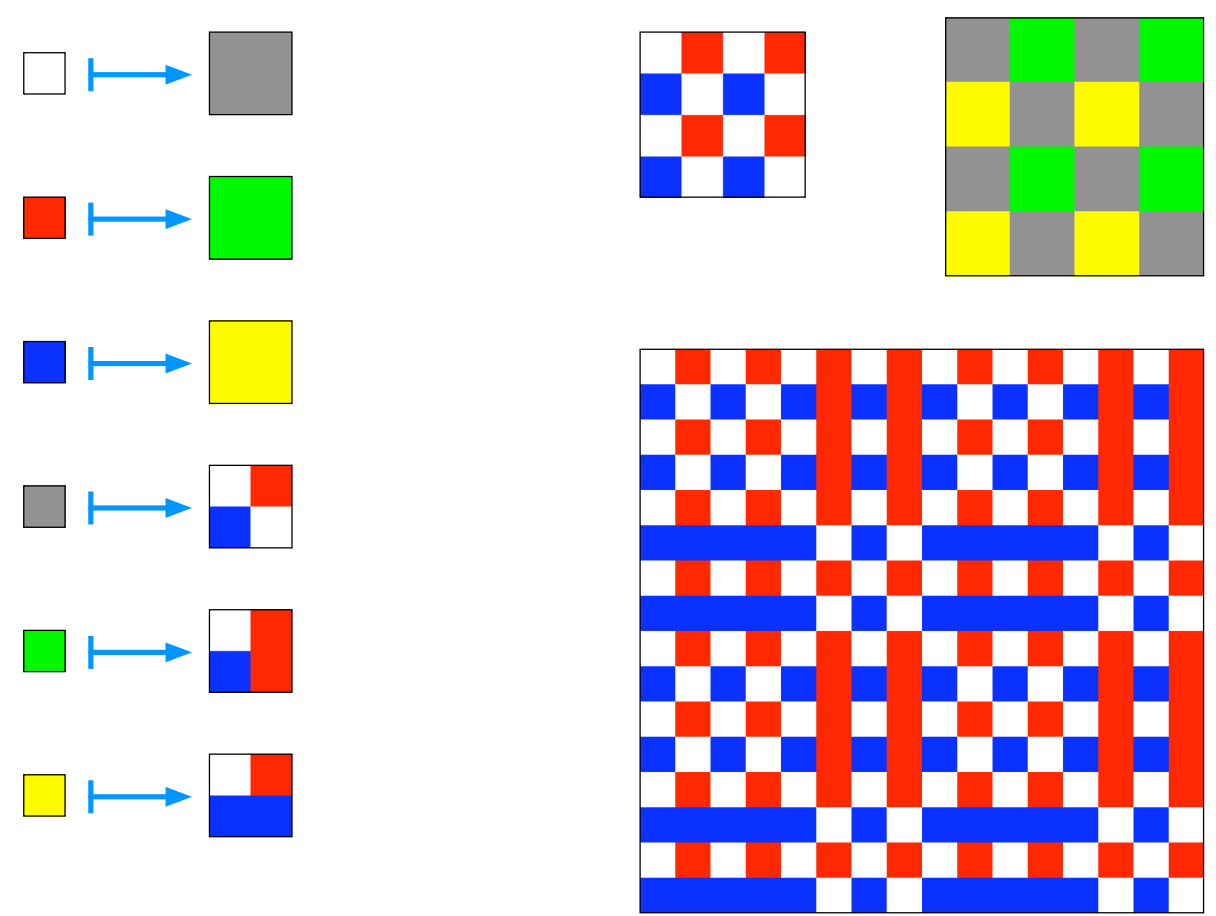

Fig. 8: Graphical s 


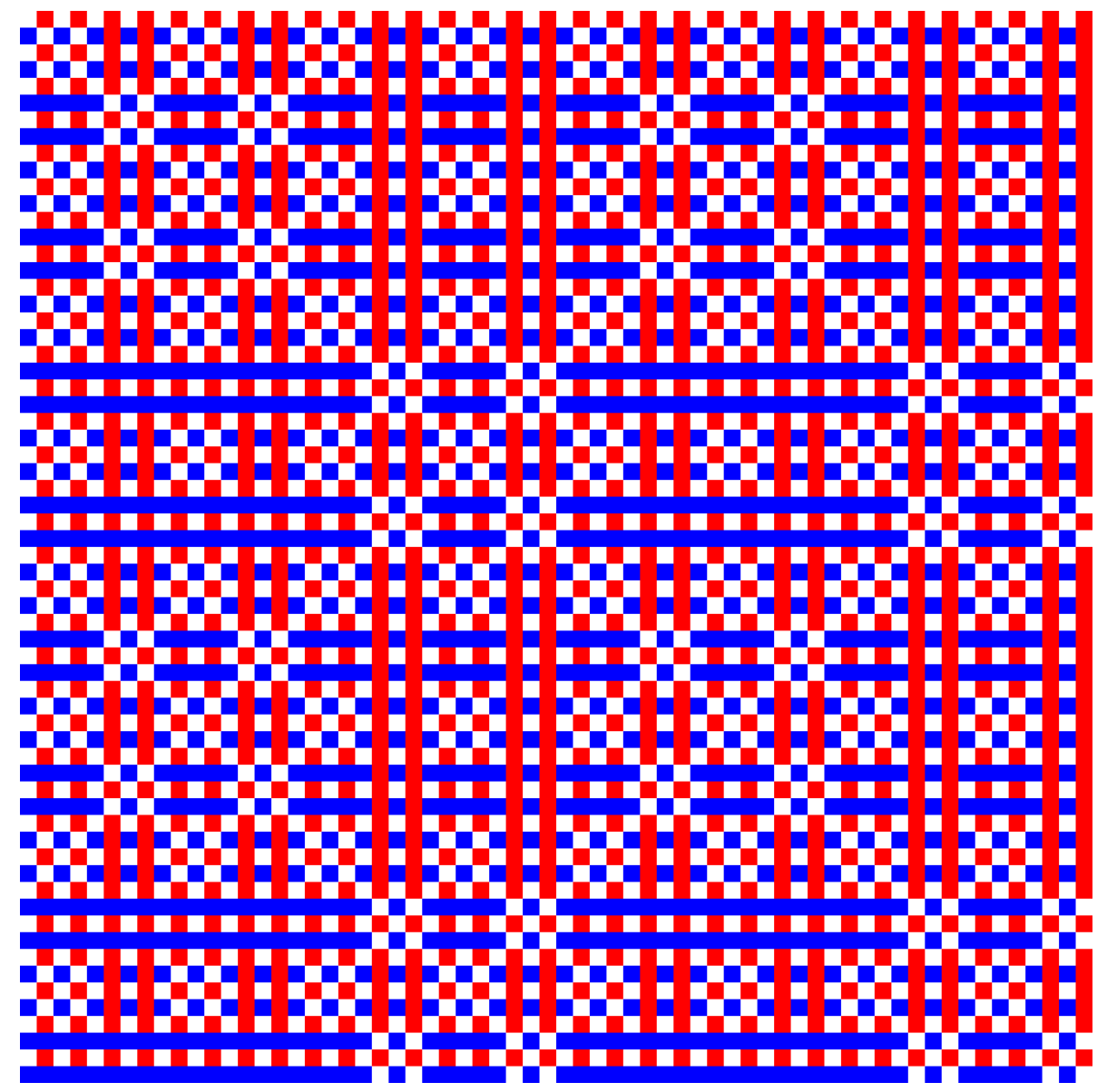

Fig. 9: Detail of $\Lambda_{\mathfrak{S}}$ 
